\title{
Spallation of Iron in Black Hole Accretion Flows
}

\author{
Jeffrey G. Skibo \\ E. O. Hulburt Center for Space Research, Code 7653, \\ Naval Research Laboratory, Washington, DC 20375-5352 \\ skibo@osse.nrl.navy.mil
}

\begin{abstract}
In the local Galactic interstellar medium there is approximate energy equipartition between cosmic rays, magnetic fields and radiation. If this holds in the central regions of AGN, in particular Seyfert galaxies, then consideral nuclear spallation of Fe occurs, resulting in enhanced abundances of the sub-Fe elements $\mathrm{Ti}, \mathrm{V}, \mathrm{Cr}$ and $\mathrm{Mn}$. These elements produce a cluster of X-ray flourescence lines at energies just below the 6.4 $\mathrm{keV} \mathrm{Fe-K \alpha}$ line. It is suggested that the red wings on the Fe lines observed with ASCA from various Seyfert AGN are due to the unresolved line emission from these elements. Future observations with more sensitive X-ray instruments should resolve these lines. The estimated gamma ray emission from nuclear deexcitation and neutral pion production is calculated and found to be below the sensitivities of any current instruments. However, very luminous nearby Seyferts displaying Fe lines with red wings would have $>100 \mathrm{MeV}$ continuum emission detectable by future instruments such as GLAST.
\end{abstract}

Subject headings: Accretion — Black Hole Physics - Cosmic Rays - Nuclear Reactions - Galaxies: Seyfert

\section{Introduction}

Recent X-ray observations with ASCA of various Seyfert AGN have revealed the presence of asymmetries in the $6.4 \mathrm{keV} \mathrm{Fe-K \alpha}$ line profile (Tanaka et al. 1995; Yaqoob et al. 1995; Mushotzky et al. 1995; Nandra et al. 1996; Iwasawa et al. 1996). The spectra generally display a narrow 6.4 $\mathrm{keV}$ line with a red wing extending below $5 \mathrm{keV}$ and a sharp cut-off above $6.5 \mathrm{keV}$. This has been interpreted as emission from the inner regions of an accretion disk of a massive black hole, where the asymmetric line profile is attributed to Doppler and gravitational effects (Tanaka et al. 1995; Fabian et al. 1995).

Other mechanisms for producing these skewed line profiles have difficulties (see Fabian et al. 1995 for a detailed discussion). For example, Comptonization is capable of producing red wings on lines, however the scattering depth required by the ASCA data is at least 3. This would 
imprint deep absorption edges in the spectrum unless the medium was nearly totally ionized. This requires the medium to be close to the central black hole, where special and general relativistic effects would dominate anyway. Another possibility is that the line is intrinsically broad and just the blue wing is absorbed. The problem is that the Fe absorption edge occurs at energies greater than $7.1 \mathrm{keV}$, whereas the ASCA data show a drop off above about $6.5 \mathrm{keV}$. Other relativistic motions not associated with accretion disks seem contrived simply because no emission to the blue is observed. However, it is also puzzling as to why in the accretion disk scenario the inferred disk orientations are always nearly face on with respect to the line-of-sight, especially in the case of NGC 4151 where the host galaxy is viewed nearly edge on (Yaqoob et al. 1995).

In this paper another scenario for producing the Fe-line red wings is introduced. Enhanced abundances of the sub-Fe metals titanium, vandium, chromium and manganese will produce flourescence lines at energies between 4.5 and $6.4 \mathrm{keV}$. The unresolved detection of these lines would appear in the spectrum as a red wing on the $6.4 \mathrm{keV}$ line of Fe. The enhancement of sub-Fe elements occurs naturally through cosmic ray spallation of Fe, the most abundant metal in astrophysical plasmas. Bombardment of an Fe nucleus by a proton of energy $\gtrsim 10 \mathrm{MeV}$ tends to chip off a light nucleus, thus converting the Fe into some isotope of an element just below Fe. Interactions of this sort will modify the abundances significantly from solar composition. This effect is observed in the relative elemental abundances of Galactic cosmic-rays, which are subject to bombardment by boosted ambient interstellar protons (Lund 1989). Lithium is also abundantly produced by spallation reactions, and excess Li has been observed in Galactic black hole systems (Martín et al. 1992).

In the local interstellar medium there is approximate energy equipartion between cosmic ray protons, ambient magnetic fields and ambient radiation fields, the energy densities are all roughly $1 \mathrm{eV} \mathrm{cm}^{-3}$. The cosmic-ray gas and ambient magnetic field are probably in approximate pressure equilibrium, but there is no known reason for the equipartition of these constituents with ambient radiation fields, it could just be coincidental. It is shown that if this situation holds in the nuclear regions of Seyfert AGN then sufficient spallation of Fe occurs resulting in the appropriate abundances of $\mathrm{Ti}, \mathrm{V}, \mathrm{Cr}$ and $\mathrm{Mn}$ for the requred 4.5-6.4 keV X-ray line emission.

In $\S 2$ nuclear spallation rates and abundances enhancements are calculated for various proton spectra. The X-ray emission resulting from the irradiation of this material by an incident power law continuum is calculated using a Monte Carlo simulation in $§ 3$. Gamma ray emission due to nuclear excition and neutral pion production is estimated in $\S 4$. Finally, a brief statement of the conclusions reached here is given in $\S 5$.

\section{Nuclear Spallation Reactions}

It is assumed that the material accreting onto the central black hole enters the accretion flow with solar composition (Anders \& Grevesse 1989) and that no further nucleosynthesis via 
fusion reactions occurs. It is also assumed that the central source produces energetic protons with luminosity approximately equal to that of the emergent radiation. This implies energy equipartition between radiation and energetic protons near the central source. No detailed assumptions concerning the geometry of the region are made. It is simply assumed that the protons are homogeniously injected into the accretion flow which provides a thick target for the protons. This is justified if the magnetic energy density is comparable that of the protons which then do not stream freely but rather have highly convoluted trajectories. This approximation is often employed in Galactic cosmic ray propagation studies and has been shown to provide an adequate explanation of the isotopic cosmic ray abundunces (Shapiro \& Silberberg 1974; Engelmann et al. 1990). In this approximation the steady state proton intensity $J_{p}(E)$ (protons $\mathrm{s}^{-1} \mathrm{~cm}^{-2}$ ster $^{-1} \mathrm{MeV}^{-1}$ ) in any local region comoving with the flow satisfies

$$
\frac{d}{d E}\left(\left|\frac{d E}{d x}(E)\right| J_{p}(E)\right)+\frac{J_{p}(E)}{\Lambda(E)}=\frac{1}{4 \pi M} \frac{d N_{p}}{d t d E}(E) .
$$

The first term on the left represents continuous energy losses due to Coulomb collisions in the ambient medium, which for the purposes here is assumed to consist of ionized hydrogen and helium with $n_{\mathrm{He}} / n_{H}=0.1$. The loss rate is approximated from the curves given in Forman, Ramaty \& Zweibel (1986):

$$
\left|\frac{d E}{d x}(E)\right|=110 E^{-0.455} \beta^{-1} \quad\left(\mathrm{MeV} \operatorname{gram}^{-1} \mathrm{~cm}^{2}\right) .
$$

The second term represents discrete energy losses due to innelastic nuclear collisions. The path length in units of grams $\mathrm{cm}^{-2}$ is given by

$$
\Lambda(E)=\frac{14 m_{p}}{10 \sigma_{p p}(E)+\sigma_{p \alpha}(E)},
$$

where $\sigma_{p p}(E)$ and $\sigma_{p \alpha}(E)$ are the inelastic $p p$ and $p \alpha$ cross sections (Meyer 1972). The term on the right is the rate per energy per accreted mass that protons are injected into the system. Here $M$ is the total mass of accretion material, not the central object. The formal solution of equation (1) is

$$
J_{p}(E)=\frac{1}{4 \pi M\left|\frac{d E}{d x}(E)\right|} \int_{E}^{\infty} d E^{\prime} \frac{d N_{p}}{d t d E}\left(E^{\prime}\right) \exp \left(-\int_{E}^{E^{\prime}} d E^{\prime \prime} \frac{1}{\left|\frac{d E}{d x}\left(E^{\prime \prime}\right)\right| \Lambda\left(E^{\prime \prime}\right)}\right)
$$

It is assumed that the injection spectrum is a simple power law in kinetic energy,

$$
\frac{d N_{p}}{d t d E}(E)=(s-2) \eta \dot{M} c^{2} E_{0}^{s-2} E^{-s},
$$

where the spectral index satisfies $s>2$. This is in accord with the source spectrum of Galactic cosmic rays inferred from measurements of the cosmic ray spectrum at the top of the atmosphere (Berezinski et al. 1990). Because the spectrum is divergent at lower energies it is necessary to cut the spectrum off below some energy $E_{0}$. The normalization in equation (5) assures a total 
proton luminosity $L_{p}=\eta \dot{M} c^{2}$ where $\eta$ represents the efficiency for converting the rest mass of the accretion flow into energetic particles. The proton luminosity is assumed to be approximately equal to the radiation luminosity where $\eta$ is generally assumed to be on the order of 0.1 .

The mean rate per target nucleus for spallation of species $i$ into species $j$ by energetic protons is given by

$$
R_{i \rightarrow j}=4 \pi \int_{0}^{\infty} d E \sigma_{i \rightarrow j}(E) J_{p}(E)
$$

where $\sigma_{i \rightarrow j}$ is the appropriate spallation cross section. In this work the empirical formulas of Letaw, Silberberg, \& Tsao (1983) are used for all spallation cross sections. Combining equations (4), (5) and (6) yields

$$
\begin{aligned}
\frac{M}{\dot{M}} R_{i \rightarrow j}=(s-2) \eta c^{2} E_{0}^{s-2} \int_{0}^{\infty} d E \sigma_{i \rightarrow j}(E) & \\
& \cdot \frac{1}{\left|\frac{d E}{d x}(E)\right|} \int_{E}^{\infty} d E^{\prime} E^{\prime-s} \exp \left(-\int_{E}^{E^{\prime}} d E^{\prime \prime} \frac{1}{\frac{d E}{d x}\left(E^{\prime \prime}\right) \mid \Lambda\left(E^{\prime \prime}\right)}\right) .
\end{aligned}
$$

The quantity on the left hand side is the spallation rate per target nucleus multiplied by the accretion time scale $t_{a} \equiv M / \dot{M}$. This is a useful quantity for what follows. The important thing is that it only depends on the input proton spectrum and the nuclear physics of spallation, and not on the details of the accretion flow.

Spallation of solar abundance material will generally deplete iron, which is the most abundant metal, and enhance the abundances of sub-Fe metals. The focus in this work is on those spallation products which produce flourescence lines in the energy range 4.5-6.4 keV. The $\mathrm{K} \alpha$ lines of titanium, vanadium, chromium and manganese are at energies 4.5, 4.9, 5.4 and $5.9 \mathrm{keV}$, respectively. The reaction network considerd here will consist of all stable isotopes of $\mathrm{Ti}, \mathrm{V}, \mathrm{Cr}$, $\mathrm{Mn}$ and Fe plus any unstable isotopes which decay into these (see Table 1).

The system of continuity equations regulating the abundances of Fe and its spallation products are

$$
\begin{aligned}
\frac{\partial N_{F e}}{\partial t} & =-N_{F e} R_{F e} \\
\frac{\partial N_{i}}{\partial t} & =-N_{i} R_{i}+N_{F e}\left(R_{F e \rightarrow i}+\sum_{j} R_{F e \rightarrow j(i)}\right)
\end{aligned}
$$

where the index $i$ extends over all stable isotopes of $\mathrm{Ti}, \mathrm{V}, \mathrm{Cr}$ and $\mathrm{Mn}$ given in the second column of Table 1. The indicies $j(i)$ in the summation extend over all unstable isotopes which decay into the stable isotope $i$. These are given in the first column of Table 1. The solutions of equations (8a) and (8b) are

$$
\begin{aligned}
N_{F e}(t) & =N_{F e}^{\odot} e^{-R_{F e} t} \\
N_{i}(t) & =N_{i}^{\odot} e^{-R_{i} t}+N_{F e}^{\odot}\left(e^{-R_{F e} t}-e^{-R_{i} t}\right)\left(\frac{R_{F e \rightarrow i}+\sum_{j} R_{F e \rightarrow j(i)}}{R_{i}-R_{F e}}\right) .
\end{aligned}
$$


The elemental abundances relative to solar values are obtained by summing over the different isotopes of each element,

$$
X_{Z}(t)=\frac{1}{N_{Z}^{\odot}} \sum_{\{A\}_{Z}} N_{A, Z}(t)
$$

where $\{A\}_{Z}$ means the sum extends over all the stable isotope listed in Table 1 belonging to element $Z$. The initial relative elemental abundances are taken from Anders \& Grevesse (1989) and the initial abundnaces of each isotope in a given element are taken from Lederer \& Shirley (1978) also given for the relevant stable end products in Table 1.

The procedure is as follows: The time scale is set equal to the accretion time scale $t_{a}=M / \dot{M}$; Equation (7) is used for the product of the spallation rates with this time scale for a given proton injection spectrum paramaterized by $s, E_{0}$ and $\eta$; Equations (9a), (9b) and (10) are then used to obtain the elemental abundances relative to solar.

In Figure 1 relative abundances are shown as a function of the proton spectral index $s$ for $E_{0}=10 \mathrm{MeV}$ and $\eta=0.1$. It can be clearly seen that the sub-Fe elements are enhanced at the expense of Fe. This is most pronounced for proton spectral indices in the range 2-3. The relative abundances depend on the assumed low energy cut-off $E_{0}$ as shown in Figure 2 where $\eta=0.1$ and $s=2.3$. Between $10-100 \mathrm{MeV}$ the dependence is rather weak until about $1 \mathrm{Gev}$ where there is generally less spallation. The dependence on the efficiency factor $\eta$ is shown in Figure 3 where $s=2.3$ and $E_{0}=10 \mathrm{MeV}$. The production of Ti, V, Cr and Mn peak at efficiencies on the order of $10 \%$.

In summary, the abundance enhancements over solar of $\mathrm{Ti}, \mathrm{V}, \mathrm{Cr}$ and $\mathrm{Mn}$ due to the spallation of Fe by energetic protons with power law spectra are greatest for proton spectral indicies in the range 2.0-2.5, low energy cut-offs between $10 \mathrm{MeV}-1 \mathrm{GeV}$ and efficiencies on the order of $10 \%$. In the following the abundance enhancements resulting from a proton spectrum with $s=2.3$, $E_{0}=10 \mathrm{MeV}$ and $\eta=0.1$ are used to calculate the X-ray line emission.

\section{X-ray Emission}

A Monte Carlo simulation is used to calculate the X-ray line emission. It is assumed that a central point source isotropically emits a simple power law X-ray continuum with energy spectral index -1 (photon spectral index -2 ). This is in accord with observations which show that the average intrinsic X-ray power law index of Seyferts is found to be close to -0.9 when the spectral hardening above $10 \mathrm{keV}$ due to reflection by cold optically thick material in the vicinity of the source is taken into account (Nandra \& Pounds 1994). However, the spectral hardening $\gtrsim 10 \mathrm{keV}$ attributed to reflection could be an intrinsic feature of the central source (Skibo \& Dermer 1995). Any hardening already present in the central source would just add slightly to the line emission,

so for simplicity a simple power law with energy index -1 is used. Material with enhanced abundances of sub-Fe elements as calculated in the last section is assumed to surround the central 
source. For simplicity, the material is assumed to have spherical geometry and uniform density. In addition to the abundances, the sphere of material is characterized by the Thomson optical depth from the center to the surface.

In the Monte Carlo simulation photons are injected at the center of the sphere with energy selected from the initial power law distribution. In the energy range considered here $(3-30 \mathrm{keV})$ the relevant processes are photoelectric absorption, flourescence line emission and Compton scattering. A photon is followed until it either scatters or is absorbed where the probabilities for these events are derived from the total cross sections. For Compton scattering the full Compton cross section is used. For photoelectric absorption the subroutines of Balucinska-Church \& McCammon (1992) are supplemented with the cross sections for the elements Ti, V and Mn taken from Henke et al. (1982). The abundances are assumed to be solar (Anders and Grevesse 1989) except for the modifications of $\mathrm{Ti}, \mathrm{V}, \mathrm{Cr}, \mathrm{Mn}$ and Fe derived in the last section. If a scattering event occurs, the photon is reinjected at the scattering location with a new direction and energy drawn from probability distributions derived from the differential Compton cross section. If the photon is absorbed the element responsible for the absorption is determined from the absorption cross sections. If the absorbing element is $\mathrm{Ti}, \mathrm{V}, \mathrm{Cr}, \mathrm{Mn}$ or $\mathrm{Fe}$ then the $\mathrm{K} \alpha$ flourescence yields $\left(Y_{T i}=0.219, Y_{V}=0.250, Y_{C r}=0.282, Y_{M n}=0.314\right.$ and $Y_{F e}=0.347 ;$ Lederer \& Shirley 1978) are used to determine whether or not a $\mathrm{K} \alpha$ line photon is emitted. If a $\mathrm{K} \alpha$ line photon is emitted then it is reinjected at the location of the absorbing event with the relevant K $\alpha$ line energy and randomly chosen direction. Ultimately, a photon escapes the system in which case it is recorded and binned, or it is absorbed without the emission of a flourescence line photon. The process is repeated until a statistically good spectrum is obtained.

In Figure 4 the emergent X-ray spectrum is shown for a reprocessing sphere of Thomson radial depth 0.1. The Ti, V, Cr, Mn and Fe abundances are those resulting from a proton spectrum with $s=2.3, E_{0}=10 \mathrm{MeV}$ and $\eta=0.1$. The $6.4 \mathrm{keV}$ Fe line is still prominant, but now with the spallation enhanced abundances, the $\mathrm{Cr}$ and $\mathrm{Mn}$ lines at $5.4 \mathrm{keV}$ and $5.9 \mathrm{keV}$, respectively, are also clearly above the continuum. There is also a hint of the $4.5 \mathrm{keV}(\mathrm{Ti})$ and $4.9 \mathrm{keV}(\mathrm{V})$ lines. The line fluxes relative to $\mathrm{Fe}$ are $4 \%(\mathrm{Ti}), 3 \%(\mathrm{~V}), 33 \%(\mathrm{Cr})$ and $11 \%(\mathrm{Mn})$ which sum to $51 \%$ of the Fe-line flux. This spectrum should not be regarded as a detailed model for the X-ray emission from Seyfert AGN. It is displayed simply to demonstrate that the lines from the sub-Fe elements will protrude above the continuum with combined strength comparable to that of the Fe-line.

Nandra et al. (1996) have produced a continuum subtracted mean line profile from the ASCA observations of a sample of 18 Seyfert 1 AGN. These data are shown in Figure 5 along with a simple model consisting of the sum of 5 gaussians each of width $0.4 \mathrm{keV}$ centered at the $\mathrm{k} \alpha$ energies of the elements $\mathrm{Ti}, \mathrm{V}, \mathrm{Cr}, \mathrm{Mn}$ and Fe. In this fit the fluxes in the gaussian lines of the sub-Fe elements relative to that of $\mathrm{Fe}$ are found to be $4 \%(\mathrm{Ti}), 10 \%(\mathrm{~V}), 33 \%(\mathrm{Cr})$ and $25 \%$ (Mn). Thus the flux in the sub-Fe elements adds up to about $72 \%$ of that in the Fe line. The data seem to require more $\mathrm{V}$ and $\mathrm{Mn}$ than that produced in the simple model presented here but otherwise are in qualitative aggreement with it. A more detailed analysis should include $\mathrm{Ni}$ and 
the secondary spallation of $\mathrm{Cr}$ which would result in more $\mathrm{V}$ and $\mathrm{Mn}$. But another possibility could be that the lines from the spalled material are intrinsically broader and that there is an additional component of narrow Fe-line emission. The important point is that, when corrections are made for the instrumental response shown in Fig 5 by the dotted curve centered at $6.4 \mathrm{keV}$, the intrinsic line widths are $0.35 \mathrm{keV}$, corresponding to velocities near 20,000 km/s. For Keplerian motion these velocities are obtained at approximately 300 Schwarzschild radii. Hence, this scenario still requires a compact nucleus, but the constraints on the size are relaxed by over an order of magnitude from those imposed by the disk-line model, which requires the emission region to be within 10 Schwarzschild radii (Tanaka et al. 1995; Fabian et al. 1995).

\section{Gamma Ray Emission}

Interactions of energetic protons will produce gamma rays. It is well known from observations of our own Galaxy that cosmic ray protons result in gamma ray continuum emission above 100 $\mathrm{MeV}$ (Bloemen 1989). Furthermore, nuclear excitation of ambient material by cosmic rays also results in nuclear line emission in the $\mathrm{MeV}$ range (Ramaty, Kozlovsky \& Lingenfelter 1996). Such line emission has been observed from Solar flares (Share \& Murphy 1995) and the Orion molecular cloud complex (Bloemen et al. 1994; Ramaty, Kozlovsky \& Lingenfelter 1995; 1996).

The continuum emission above $100 \mathrm{MeV}$ is produced mainly by strong interactions of protons with ambient nuclei resulting in neutral pions which subsequently decay into photons. The gamma ray production is calculated using the cross sections and codes developed by Dermer (1986). Using the form for the proton spectrum given in equation (5) the gamma ray emission is given by

$$
\begin{aligned}
& \frac{d N_{\gamma}}{d t d \epsilon}(\epsilon)=\dot{N}(s-2) \eta c^{2} E_{0}^{s-2} \int_{0}^{\infty} d \epsilon \frac{d \sigma_{p H \rightarrow \gamma^{\prime} s}}{d \epsilon}(\epsilon, E) \\
& \cdot \frac{1}{\left|\frac{d E}{d x}(E)\right|} \int_{E}^{\infty} d E^{\prime} E^{\prime-s} \exp \left(-\int_{E}^{E^{\prime}} d E^{\prime \prime} \frac{1}{\left|\frac{d E}{d x}\left(E^{\prime \prime}\right)\right| \Lambda\left(E^{\prime \prime}\right)}\right) .
\end{aligned}
$$

where $\dot{N}=\dot{M} / m_{p}$ is the baryon accretion rate. Using parameters typical of Seyferts and the proton spectrum with $s=2.3, E_{0}=10 \mathrm{MeV}$ and $\eta=0.1$, the integrated $>100 \mathrm{MeV}$ gamma ray flux at earth is estimated to be

$$
\operatorname{Flux}(>100 \mathrm{MeV}) \simeq 10^{-7}\left(\frac{L}{10^{44} \mathrm{erg}}\right)\left(\frac{d}{1 \mathrm{Mpc}}\right)^{-2} \quad\left(\text { photons s}^{-1}\right) .
$$

This falls below the $2 \sigma$ flux upper limits of Seyferts obtained with EGRET (Lin et al. 1993), but not by much. A very luminous $\left(z 10^{44}\right)$ nearby Seyfert displaying the skewed Fe line profile could be observable at high energies with the proposed GLAST instrument if indeed the red wing on the Fe line is due to the unresolved line emission from sub-Fe elements.

The nuclear deexcitation line emission is estimated from the yields per proton calculated for 
solar flares (Murphy 1985). The emission rate of narrow nuclear line photons is approximately

$$
\frac{d N_{\gamma}}{d t} \simeq 10^{-3} \int_{30 \mathrm{MeV}}^{\infty} d E \frac{d N_{p}}{d t d E}(E)
$$

For the proton spectrum assumed in this analysis the line flux at earth is estimated to be

$$
\text { Flux }(\text { narrow lines }) \simeq 10^{-6}\left(\frac{L}{10^{44} \mathrm{erg}}\right)\left(\frac{d}{1 \mathrm{Mpc}}\right)^{-2} \quad\left(\text { photons s}^{-1}\right) .
$$

Again, this is well below the sensitivities of any instruments ever built. This also falls below the sensitivity of the planned low energy gamma ray spectrometer INTEGRAL.

\section{Conclusion}

The assumption of approximate energy equipartition between energetic protons, magnetic

fields and radiation in the central regions of Seyfert AGN implies that consideral nuclear spallation of Fe must occur in the accretion flow. This results in enhanced abundances relative to solar of the sub-Fe elements $\mathrm{Ti}, \mathrm{V}, \mathrm{Cr}$ and $\mathrm{Mn}$. The $\mathrm{K} \alpha$ line emission of these elements provides an explanation for the asymmetries on the $6.4 \mathrm{keV}$ Fe-K $\alpha$ lines observed from various Seyferts with ASCA. The energetic protons also produce gamma ray line and continuum emission from nuclear deexcitation and neutral pion production. This emission is estimated to be below the sensitivities of current gamma ray instruments, however, very luminous nearby Seyferts displaying Fe-line red wings could be observed by future instruments such as GLAST.

I thank C. H. Tsao for providing subroutines for the spallation cross sections, Chuck Dermer for his pion code, Bernard Phlips, Ron Murphy, Brad Graham, Greg Jung and Tahir Yaqoob for useful discussions.

\section{REFERENCES}

Anders, E. \& Grevesse, N. 1989, Geochim. Cosmochim. Acta, 53, 193

Balucińska-Church, M. \& MacCammon, D. 1982, ApJ, 400, 699

Berezinskii, V. S. et al. 1990, Astrophysics of Cosmic Rays, (North Holland: Amsterdam)

Bloemen, H. 1989, ARA\&A, 27, 469

Bloemen, H. et al. 1994, A\&A, 281, L5

Dermer, C. D. 1986, A\&A, 157, 223

Engelmann, J. J. et al. 1990, A\&A, 233, 96

Fabian, A. C., Nandra, K., Reynolds, C. S., Brandt, W. N., Otani, C., Tanaka, T., Inoue, H., \& Iwasawa, K. 1995, MNRAS, 277, L11 
Forman, M. A., Ramaty, R. \& Zweibel, E. G. 1986, Physics of the Sun, ed P. A. Sturrock, (Reidel), 249

Henke, B. L., Lee, P., Tanaka, T. J., Shimabukuro, R. L., \& Fujikawa, B. K. 1982, Atomic Data Nuclear Data, 27, 1

Iwasawa, K. et al. 1996, MNRAS, in press

Lederer, C. M. \& Shirley, V. S. 1978, Table of Isotopes (Wiley and Sons: New York)

Letaw, J. R., Silberberg, R., \& Tsao, C. H. 1983, ApJS, 51, 271

Lin, Y. C. et al. 1993, ApJ, 416, L53

Lund, N. 1989, Cosmic Abundances of Matter, ed. C. J. Waddington (AIP: New York), 111

Martín, E. L., Rebolo, R., Casares, J., \& Charles, P. A. 1992, Nature, 358, 129

Meyer, J. P. 1972, A\&AS, 7, 417

Murphy, R. 1985, Ph.D. Thesis, University of Maryland

Mushotzky, R. F., Fabian, A. C., Iwasawa, K., Kunieda, H., Matsuoka, M., Nandra, K., Tanaka, Y. 1995, MNRAS, 272, L9

Nandra, K., George, I. M., Mushotzky, R. F., Turner, T. J., \& Yaqoob, T. 1996, ApJ, in press

Nandra, K. \& Pounds, K. A. 1994, MNRAS, 268, 405

Ramaty, R., Kozlovsky, B., \& Lingenfelter, R. E. 1995, ApJ, 438, L21

Ramaty, R., Kozlovsky, B., \& Lingenfelter, R. E. 1996, ApJ, 456, 525

Shapiro, M. M. \& Silberberg, R. 1974, Phil. Trans. Roy. Soc., 227, 319

Share, G. H. \& Murphy, R. J. 1995, ApJ, 452, 933

Skibo, J. G. \& Dermer, C. D. 1995, ApJ, 455, L25

Tanaka, Y. et al. 1995, Nature, 375, 659

Yaqoob, T., Edelson, R., Weaver, K. A., Warwick, R. S., Mushotzky, R. F., Serlemitsos, P. J., \& Holt, S. S. 1995, ApJ, 453, 81 
Table 1. Spallation Products of Fe

\begin{tabular}{lc}
\hline \hline Unstable Products & $\begin{array}{c}\text { Stable End Products } \\
\text { (Natural Isotopic Abundance Percentages) }\end{array}$ \\
\hline${ }^{46} \mathrm{~V},{ }^{46} \mathrm{Cr}$ & ${ }^{46} \mathrm{Ti}(8.2)$ \\
${ }^{47} \mathrm{~V},{ }^{47} \mathrm{Cr}$ & ${ }^{47} \mathrm{Ti}(7.4)$ \\
${ }^{48} \mathrm{~V},{ }^{48} \mathrm{Cr}$ & ${ }^{48} \mathrm{Ti}(73.7)$ \\
${ }^{49} \mathrm{~V},{ }^{49} \mathrm{Cr}$ & ${ }^{49} \mathrm{Ti}(5.4)$ \\
$\ldots$ & ${ }^{50} \mathrm{Ti}(5.2)$ \\
$\ldots$ & ${ }^{50} \mathrm{~V}(0.25)$ \\
${ }^{50} \mathrm{Mn}$ & ${ }^{50} \mathrm{Cr}(4.35)$ \\
${ }^{51} \mathrm{Ti},{ }^{51} \mathrm{Cr}$ & ${ }^{51} \mathrm{~V}(99.75)$ \\
${ }^{52} \mathrm{Mn}$ & ${ }^{52} \mathrm{Cr}(83.8)$ \\
${ }^{53} \mathrm{Mn}$ & ${ }^{53} \mathrm{Cr}(9.5)$ \\
${ }^{54} \mathrm{Mn}$ & ${ }^{54} \mathrm{Cr}(2.36)$ \\
${ }^{55} \mathrm{Fe}$ & ${ }^{55} \mathrm{Mn}(100)$ \\
\hline
\end{tabular}


Fig. 1 - The abundances of Ti, V, Cr, Mn and Fe relative to their values in the solar photosphere (Anders \& Grevesse 1989) versus the spectral index of the power law proton injection spectrum. The proton spectrum is cut off below $E_{0}=10 \mathrm{MeV}$ and the efficiency of converting the accretion mass into energetic protons is $\eta=0.1$.

Fig. 2 - The abundances of Ti, V, Cr, Mn and Fe relative to their values in the solar photosphere (Anders \& Grevesse 1989) versus the low energy cut off of the power law proton injection spectrum. The proton spectral index is $s=2.3$ and the efficiency of converting the accretion mass into energetic protons is $\eta=0.1$.

Fig. 3 - The abundances of Ti, V, Cr, Mn and Fe relative to their values in the solar photosphere (Anders \& Grevesse 1989) versus the efficiency of converting the accretion mass into energetic protons. The protons are cut off below $E_{0}=10 \mathrm{MeV}$ and the proton spectral index is $s=2.3$.

Fig. 4 - The X-ray spectrum resulting from a Monte Carlo simulation of the emission from a central source with power law spectrum (energy index -1) passing through a sphere of Thomson scattering depth 0.1. The abundances are solar except for Ti, V, Cr, Mn and Fe which are modified by the linear factors: $9.2(\mathrm{Ti}) ; 53(\mathrm{~V}) ; 15(\mathrm{Cr}) ; 10(\mathrm{Mn})$; and $0.48(\mathrm{Fe})$. The dotted histogram is the spectrum of the central source normalized to 1 photon and the solid histogram is the reprocessed spectrum.

Fig. 5 - The data represent the continuum subtracted mean line profile for a sample of 18 Seyfert 1 AGN observed with the SIS on ASCA (Nandra et al 1996). The solid curve is the sum of 5 gaussians each of width $0.4 \mathrm{keV}$ centered at the $\mathrm{K} \alpha$ energies of the elements $\mathrm{Ti}, \mathrm{V}, \mathrm{Cr}, \mathrm{Mn}$ and Fe. The fluxes in the gaussian lines of the sub-Fe elements relative to that of Fe are $4 \%$ (Ti), $10 \%$ $(\mathrm{V}), 33 \%(\mathrm{Cr})$ and $25 \%(\mathrm{Mn})$. The instrumental response is shown by the dotted curve centered at $6.4 \mathrm{keV}$. 


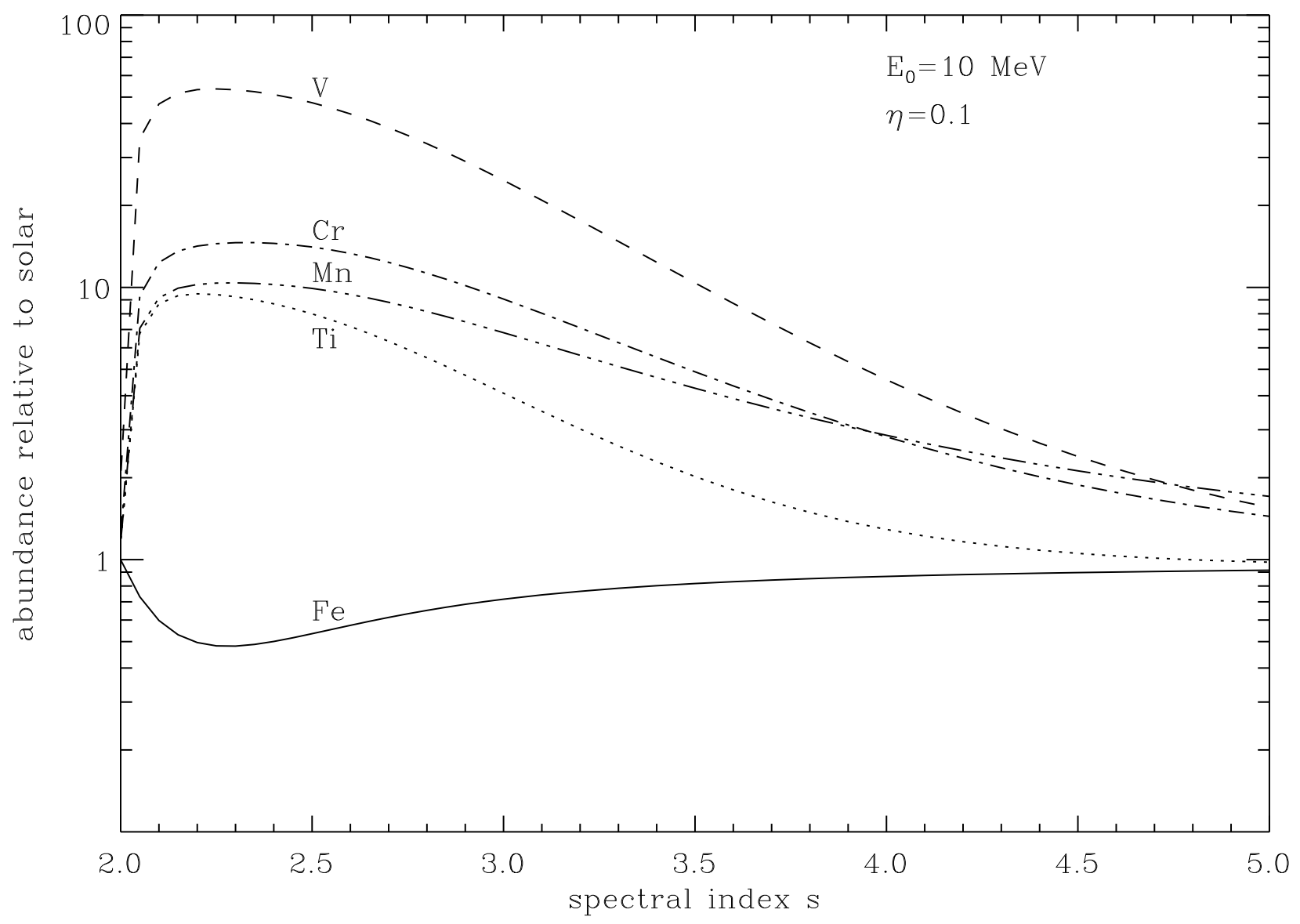

Figure 1 


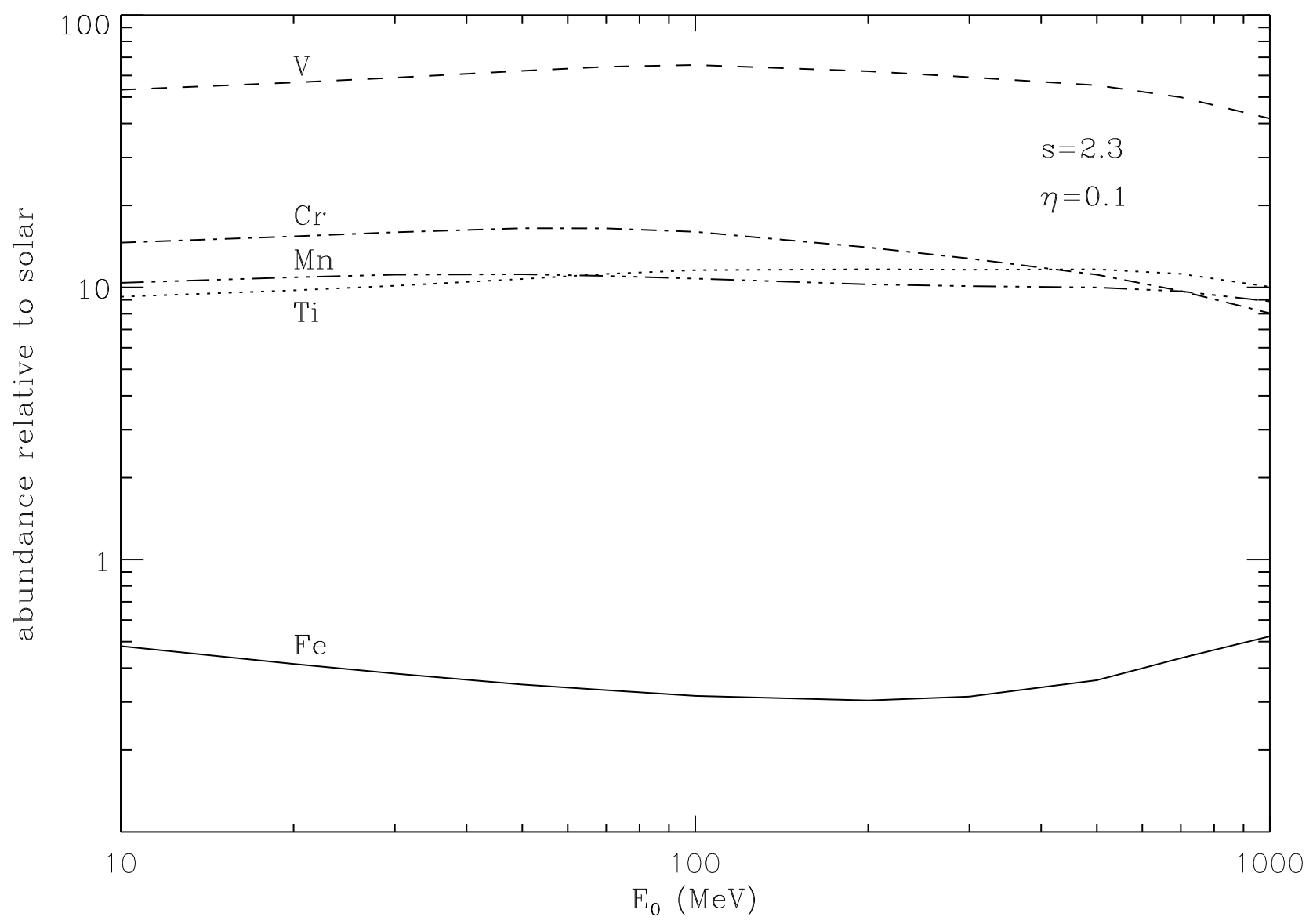

Figure 2 


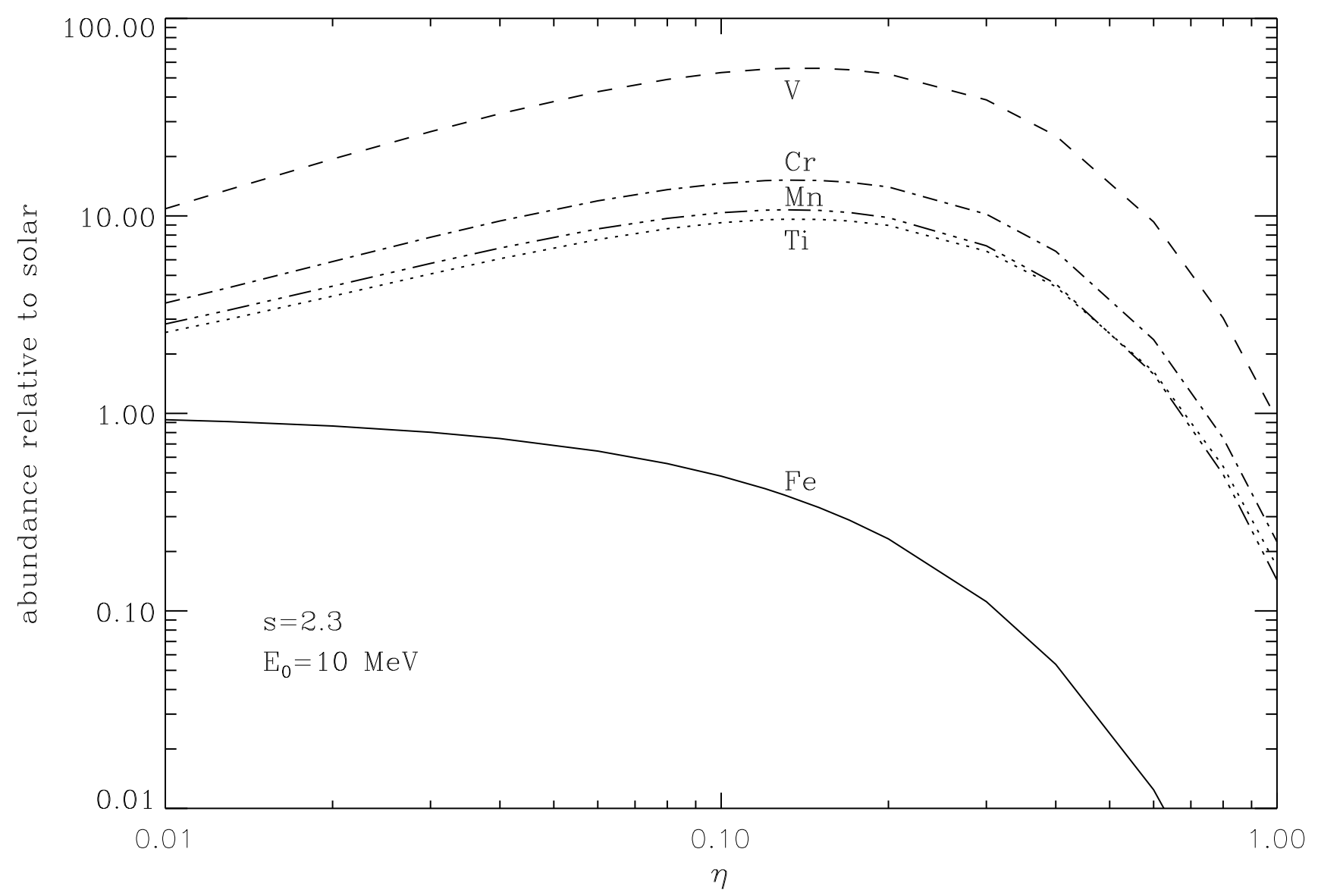

Figure 3 


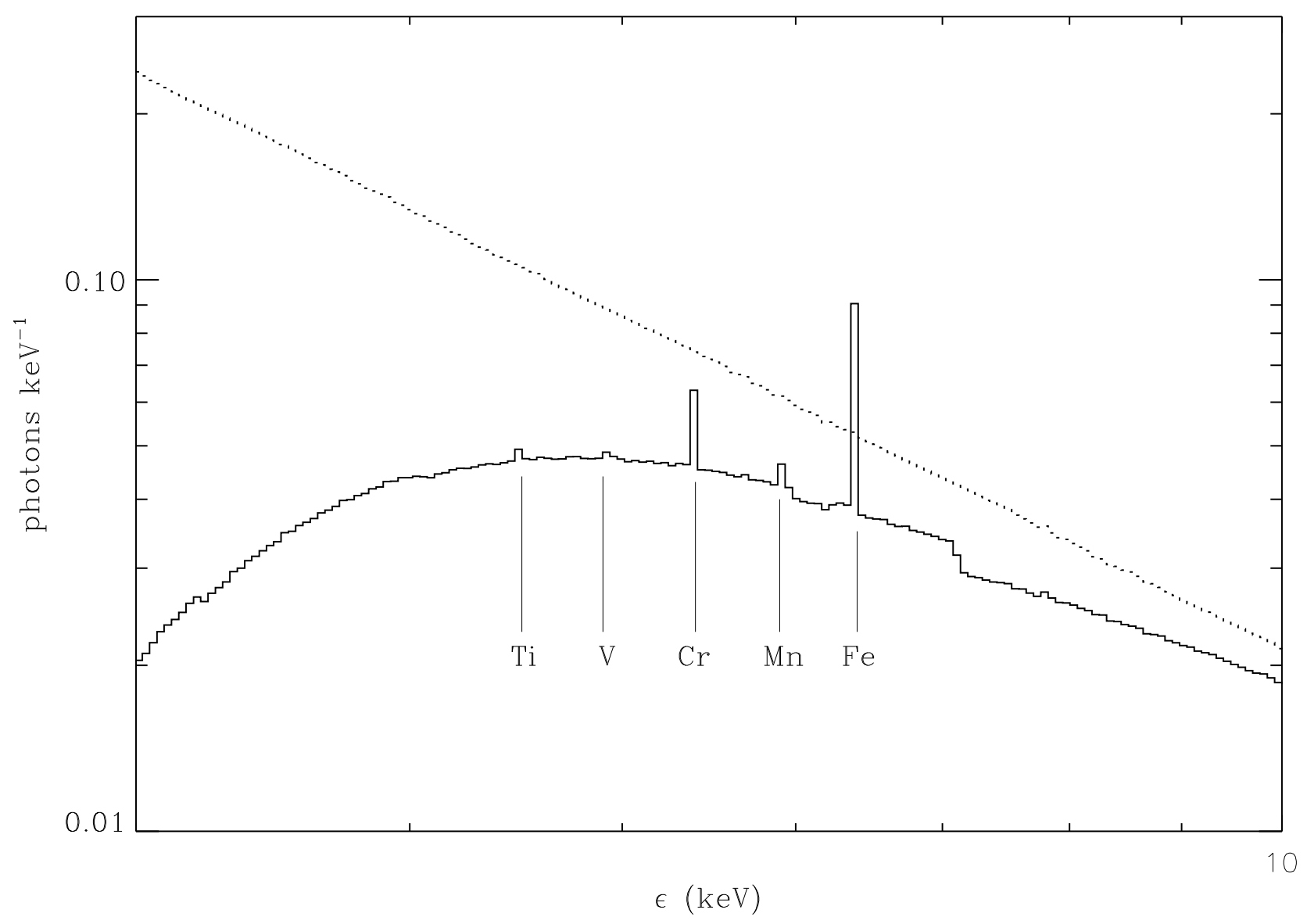

Figure 4 


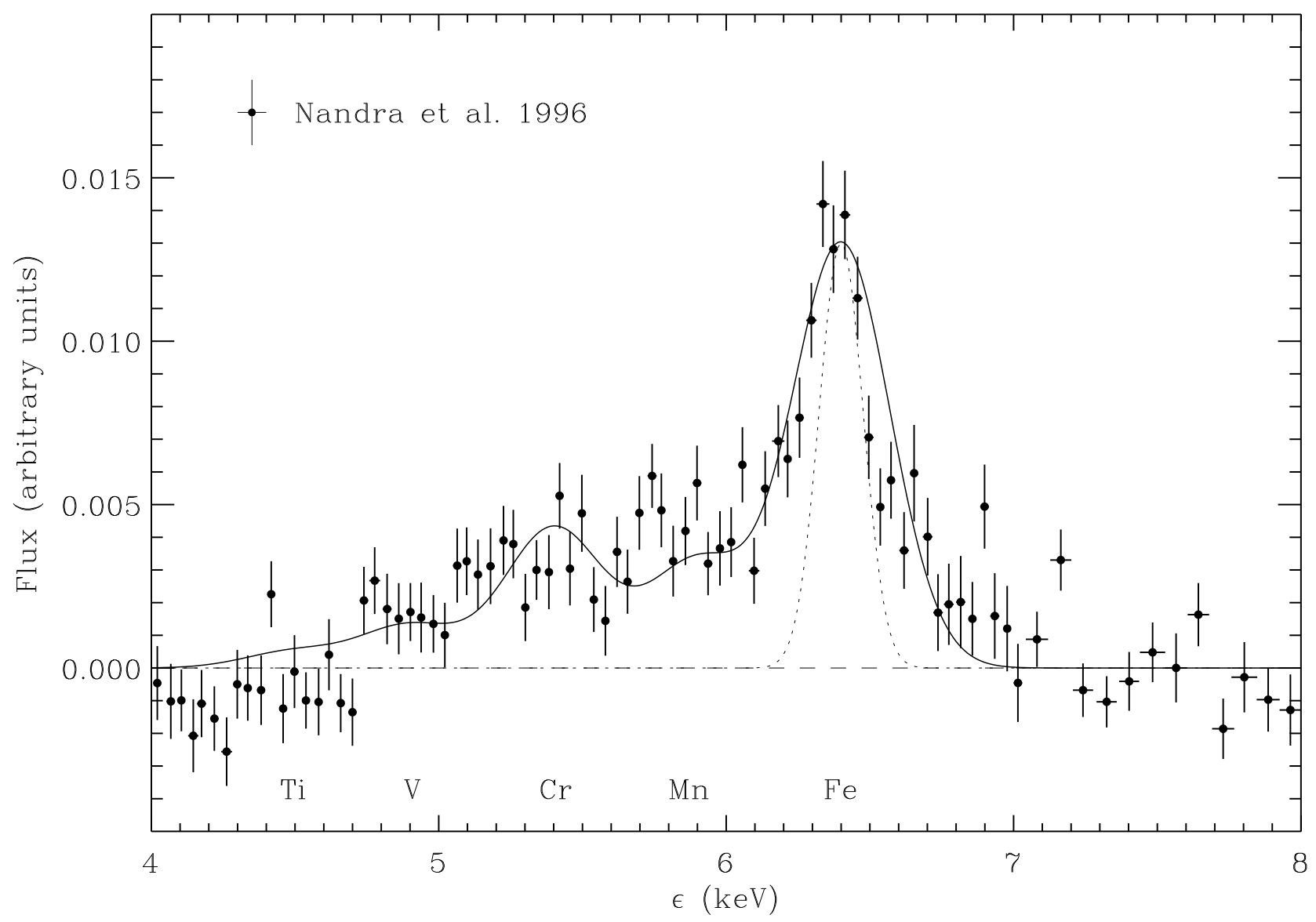

Figure 5 